\title{
Iron - introduction to a short series of articles
}

\author{
PAWEe LIPIŃSKI \\ Department of Molecular Biology, Institute of Genetics and Animal Breeding \\ Polish Academy of Sciences, Jastrzębiec, Poland \\ e-mail: p.lipinski@ighz.pl
}

The Earth has a liquid outer and a solid inner core that are predominantly composed of iron. Iron is the second most abundant metal of the Earth's crust. For reasons of the geochemical ubiquity of $\mathrm{FeS}$ and the catalytic roles of iron and sulfur at the origin of first biomolecules and organisms, the chemo-autotrophic hypothesis reconstructing the beginnings of life on Earth has been proposed and dubbed the "Iron-Sulfur-World" theory. Iron is also ubiquitous in organic systems. The high abundance and unique redox properties of iron, prompted it to be largely used in vital biological processes. The bioselection of iron continued even when dioxygen $\left(\mathrm{O}_{2}\right)$ evolved into the Earth's atmosphere. Neither iron oxidation and its transformation into the insoluble $\mathrm{Fe}(\mathrm{III})$ state nor its apparent toxic interaction with oxygen-derived species excluded iron from the biological processes. For the vast majority of living organisms, iron is absolutely necessary for the maintenance, defense, differentiation, growth and cellular division. Because of its biological indispensability and potential toxicity, iron is often called a double-edged sword or a metal with a dual nature. It is therefore not surprising that in all organisms, iron homeostasis relies on two simultaneously occurring processes - the maintenance of iron bioavailability and the limitation of iron toxicity.

Within the past 15 years, an enormous progress has been made in the understanding of the molecular basis of mammalian iron homeostasis. Many of the key proteins involved in iron homeostasis have been identified and characterized. It is nowadays difficult to believe that as recently as in the mid nineties of the past century iron metabolism was mainly perceived through the functions of ferritin, transferrin and transferrin cell membrane receptor. All these achievements in the understanding of body iron balance have provided important insights into the pathophysiology of human iron disorders. The identification in 1997 of $H F E$, a candidate gene for genetic hemochromatosis, an autosomal recessive disease and one of the most frequent genetic disorders in man, has allowed molecular diagnosis and strongly contributed to the identification of other genes involved in this iron-related disease. A recent discovery of liver-derived peptide hepcidin and its role in the concerted regulation of iron release from absorptive enterocytes and macrophages through its interaction with ferroportin has proved to be fundamental in the understanding of iron circulation and allocation in the body. Furthermore, the hepcidin-ferroportin axis appears to be a promising target for the diagnosis and treatment of both, iron overload and iron deficiency disorders. Importantly, the knowledge of iron biology makes us realize that iron homeostasis is not an issue per se. In this context, it is worth recalling that a disturbed iron balance leads to common hematological, metabolic, immunological, and neurodegenerative diseases.

During the Launch Meeting of Polish Iron Club (PIC) held at the Institute of Genetics and Animal Breeding of the Polish Academy of Sciences in Jastrzębiec last June, an initiative emerged to provide the milieu of Polish biologists with recent advances in iron metabolism through the cycle of concise reviews published in BioTechnologia. In the present and coming issues of BioTechnologiawe are going to present the readers with an outline of cellular and systemic iron regulation in mammals. We will show how mathematical models may be of help in delineating the complex regulatory networks of iron homeostasis. We explore the role of $H F E$ gene mutations in the pathogenesis of hemochromatosis, the most common inherited iron overload disorder (occurring frequently also in Poland). The paper that discusses the role of iron in neurodegenerative disorders such as Parkinson disease shed light on the perturbation of iron homeostasis in the brain, an important, but still an incompletely understood issue. The next paper that 
refers to iron deficiency anemia in obese people raises the importance of the interaction between iron, "an ancient micronutrient" and obesity, "a modern disease". Finally, the negative effects coming from the interactions between iron and oxygen are exemplified in the paper showing association between body iron stores and oxidative damage to DNA.

Iron is a hot topic all over the world. The turn of the $20^{\text {th }}$ century is called the "golden age" of iron biology. Surprisingly, the contribution of Polish researchers into the field of iron biology is rather modest and sharply contrasts with the extensive achievements obtained in European and American laboratories. We hope that this series of papers, together with other activities such as the foundation of PIC and the organization of "the iron session" during The Congress of Biochemistry and Cell Biology in Cracow (September 2011), will serve as a "magnet" attracting interest of Polish scientists in the biological and medical roles of iron. 